\title{
Design and Implementation of Combined Energy Metric AODV (CEM_AODV) Routing Protocol for MANETs
}

\author{
Annapurna P. Patil, Bathey Sharanya, M. P. Dinesh Kumar, and Malavika J.
}

\begin{abstract}
Mobile Ad Hoc Networks (MANETs) also called mesh networks are self-configuring networks of mobile devices connected by wireless links. MANETs are deployed in situations where there is no existing infrastructure, such as emergency search and rescue, military, battlefields, etc. Each application has a different set of requirements. In this paper we concentrate on Emergency search and rescue operations which rely heavily on the availability of the network. The availability is a direct cost of the overall network lifetime, i.e., energy of the nodes. There are many strategies available at different levels of the OSI model to improve the network lifetime. The focus is on developing a network layer strategy, i.e., one that uses routing protocols. AODV protocol is seen to be the most energy efficient protocol.

Firstly we select two existing energy efficient routing protocols based on AODV, each of which is based on a different energy cost metric. And then design a protocol that is a combination of both, hence a combination of two energy cost metrics. Secondly we evaluate the performance of this protocol against the single energy metric AODV protocol and against traditional AODV. The performance metrics used for evaluation are packet delivery ratio, throughput, convergence time, network lifetime and average energy consumed. The simulation is done using NS2 network simulator.
\end{abstract}

Index Terms-AODV, energy efficiency, MANETs, NS2.

\section{INTRODUCTION}

Mobile ad hoc networks (MANETs) are composed of a collection of mobile nodes which can move freely and communicate with each other using a wireless physical medium without having to resort to a pre-existing infrastructure. Therefore, dynamic topology, unstable links, limited energy capacity and absence of fixed infrastructure are special features for MANET when compared to wired networks. MANET does not have centralized controllers, which makes it different from traditional wireless networks (cellular networks and wireless LAN) [1].

MANETs, find applications in several areas. Some of them are: military applications, collaborative and distributed computing, emergency operations, wireless mesh networks, wireless sensor network, and hybrid wireless network architectures [2].

Manuscript received October 9, 2012; revised November 23, 2012.

Annapurna P. Patil with the National Institute of Standards and Technology, Boulder, CO 80305 USA (e-mail: author@boulder.nist.gov).

Bathey Sharanya is was with Rice University, Houston, TX 77005 USA. $\mathrm{He}$ is now with the Department of Physics, Colorado State University, Fort Collins, CO 80523 USA (e-mail: author@lamar. colostate.edu).

M. P. Dinesh Kumar is with the Electrical Engineering Department, University of Colorado, Boulder, CO 80309 USA, on leave from the National Research Institute for Metals, Tsukuba, Japan (e-mail: author@nrim.go.jp).
Energy is a scarce resource in ad hoc wireless networks [3]. Each node has the functionality of acting as a router along with being a source or destination. Thus the failure of some nodes operation can greatly impede performance of the network and even affect the basic availability of the network, i.e., routing, availability, etc. Thus it is of paramount importance to use it efficiently when establishing communication patterns. Energy management is classified into battery power management, transmission power management, system power management [2].

In recent years, a number of studies have been done in different layers, such as MAC layer and application layer, of the OSI model to achieve energy conservation. Our work focuses only on the routing/network layer. The characteristics of MANETs have led to the design of MANET specific routing protocols. These protocols are mainly classified as proactive and reactive [2]. Proactive protocols are table driven i.e., nodes maintain information regarding the routes. Reactive routing protocol find the routes only when they are needed i.e., on-demand. Routing protocols without consideration of energy consumption tend to use the same paths and exhaust the nodes. The reactive routing protocols and in particular Ad hoc On-demand Distance Vector (AODV) is found to be the most energy efficient [1], [4]. There are four energy cost metrics based on which we can decide the energy efficiency of a routing protocol. They are transmission power, remaining energy capacity, estimated node lifetime and combined energy metrics. Also a combination of the energy metrics specified above is proven to be more efficient than one metric alone [1]. Hence our work is mainly concentrated towards improving the existing AODV algorithm, using two energy cost metrics, to obtain an energy efficient AODV algorithm.

The paper is organized as follows: In section II, we briefly discuss the literature related to our paper. In section III, we briefly discuss the related work. Section IV, provides a detail description of the design and implementation. Section V shows the results. Section VI discusses the conclusion and Section VII gives the future work.

\section{LITERARURE SURVEY}

The design of an energy efficient routing protocol for MANETs requires a detailed insight into routing and energy management strategies for MANETs.

The characteristics of MANETs have led to the development of MANET specific routing protocols. A routing protocol is the mechanism by which user traffic is directed and transported through the network from a source node to a destination node. Based on this definition the 
classification of routing protocols is given as follows.

\section{A. Classification of Routing in MANETs}

MANET routing protocols could be broadly classified into two major categories based on the routing information update mechanism [5]:

1) Proactive Routing Protocols: Proactive protocols continuously learn the topology of the network by exchanging topological information among the network nodes. Ex: DSDV, WRP, CGSR, etc.

2) Reactive Routing Protocols: The reactive routing protocols are based on some sort of query-reply dialog. Ex: DSR, AODV, TORA, etc.

3) Hybrid Routing Protocols: Often reactive or proactive feature of a particular routing protocol might not be enough; instead a mixture might yield better solution. Hence, in the recent days, several hybrid protocols are also proposed.

Proactive approaches introduce more overhead compared to reactive ones. This is because even when there are no changes in network topology, control messages are flooded in order to maintain a full knowledge of the network in each node [6]. In proactive routing protocols first packet latency is less when compared with on-demand protocols [6]. Proactive (Table-driven) protocols are inherently more energy consuming compared to Reactive (On-demand) ones, hence most of the proposals involve modifications to reactive protocols [5]. In Reactive protocols, AODV is found to be the most energy efficient routing protocol [7], therefore most have the studies concentrate on making AODV routing protocol more energy efficient.

\section{B. Ad hoc On-Demand Distance Vector}

AODV [5] is a reactive routing protocol instead of proactive. It minimizes the number of broadcasts by creating routes based on demand, which is not the case for DSDV. When any source node wants to send a packet to a destination, it broadcasts a route request (RREQ) packet. The neighbouring nodes in turn broadcast the packet to their neighbours and the process continues until the packet reaches the destination. During the process of forwarding the route request, intermediate nodes record the address of the neighbour from which the first copy of the broadcast packet is received. This record is stored in their route tables, which helps for establishing a reverse path. If additional copies of the same RREQ are later received, these packets are discarded. The reply is sent using the reverse path. For route maintenance, when a source node moves, it can reinitiate a route discovery process. If any intermediate node moves within a particular route, the neighbour of the drifted node can detect the link failure and sends a link failure notification to its upstream neighbour. This process continues until the failure notification reaches the source node. Based on the received information, the source might decide to re-initiate the route discovery phase.

Energy management in MANETs is the basis on which routing protocols are improved to attain energy efficiency. The choice of the routing protocol affects each of the dimensions along which energy is consumed, such as transmission, battery, device, processor energy. These dimensions are discussed in detail in the remainder of the section. Along with these schemes there is also a description of the energy cost metrics which measure the amount of energy saved by using different path selection schemes.

\section{Energy Management in MANETs}

Energy is a scarce resource in ad hoc wireless networks and it is of paramount importance to use it efficiently when establishing communication patterns [3].

Energy Management is defined as the process of managing the sources and consumers of energy in a node or in a network as a whole for enhancing the lifetime of the network [2].

Energy Management can be classified into the following categories [2]: transmission power management, battery energy management, processor power management, devices power management.

\section{Energy Efficiency Metrics}

A survey of the recent research in energy efficient routing protocols for ad hoc networks allows classifying the power efficient routing protocols into four categories based on their path selection scheme.

The first set of protocols use the energy cost for transmission as the cost metric and aim to save energy consumption per packet. However, such protocols do not take the nodes' energy capacity into account. Thus, the energy consumption is not fair among nodes in the network.

Minimum Total Transmission Power Routing (MTPR) [1] is an example protocol for this category. The second set of protocols use the remaining energy capacity as the cost metric, which means that the fairness of energy consumption becomes the main focus. But, these protocols cannot guarantee the energy consumption is minimized. The third set of protocols is similar to the second set, but use estimated node lifetime instead of node energy capacity as the route selection criteria. Therefore, these protocols still aim to fairly distribute energy consumption. In order to both conserve energy consumption and achieve consumption fairness, Conditional Max-Min Battery Capacity Routing (CMMBCR) [1] has been proposed to combine these two metrics. CMMBCR is an example of the fourth category of protocols, which use combined metrics to represent energy cost.

\section{RELATED WORK}

The proposed work is aimed at developing an energy efficient AODV routing protocol therefore this section studies some of the many energy efficient schemes using AODV algorithm developed by researchers in the field.

In [8], Jin-Man Kim and Jong-Wook Jang proposes an enhanced AODV (Ad-hoc On-demand Distance Vector) routing protocol which is modified to improve the networks lifetime in MANET (Mobile Ad-hoc Network). One improvement for the AODV protocol is to maximize the networks lifetime by applying an Energy Mean Value algorithm which considerate node energy-aware. Increase in the number of applications which use Ad hoc network has led to an increase in the development of algorithms which consider energy efficiency as the cost metric.

In [9], Yumei Liu, Lili Guo, Huizhu Ma and Tao Jiang 
propose a multipath routing protocol for mobile ad hoc networks is proposed, called MMRE-AOMDV, which extends the Ad Hoc On-demand Multipath Distance Vector (AOMDV) routing protocol. The key idea of the protocol is to find the minimal nodal residual energy of each route in the process of selecting path and sort multi-route by descending nodal residual energy. Once a new route with greater nodal residual energy is emerging, it is reselected to forward rest data packets. It can balance individual node's battery power utilization and hence prolong the entire network's lifetime.

In [10], Zhang Zhaoxiao, Pei Tingrui and Zeng Wenli propose a new mechanism of energy-aware named EAODV for Ad Hoc is proposed in this paper, which is based on the classical AODV (the routing protocol on demand). And backup routing mechanism is adopted. In EAODV, the route which spends less energy and owns larger capacity is selected by synthetic analysis.

Therefore from the research work done many proposals for optimizing AODV to make it energy efficient were seen. In the next section we propose the method to optimize AODV by combining two energy cost metrics.

\section{DESIGN AND IMPLEMENTATION}

The algorithm which we propose integrates two energy metrics into AODV in an efficient way so that the Ad hoc network has a greater life time and energy consumption across the nodes is reduced. The two energy metrics which we try to combine are:

1) Transmission Power

2) Remaining Energy Capacity

Here, for each metric used by certain routing protocols, we always consider a $k$-hop route $R=v 0, v 1, \ldots$, vk from the source $v 0$ to destination $v k$. We also use the following notations:

TABLE I: EXPLANATION OF THE NOTATIONS

\begin{tabular}{|l|l|}
\hline Notations & Meaning \\
\hline$C_{R}$ & Cost of route $R$ \\
\hline$P_{T}(i)$ & Transmission power of node $v_{i}$ \\
\hline$P_{R}(i)$ & Receiving power of node $v_{i}$ \\
\hline$E_{r}^{i}(t)$ & Remaining energy capacity of node $v_{i}$ at time $t$ \\
\hline$E_{o}^{i}$ & Initial energy capacity of node $v_{i}$ \\
\hline
\end{tabular}

\section{A. Transmission Power}

The received signal power attenuates as $d^{-n}$ where $d$ is the transmission distance, and usually, $n=2$ for short distance and $n=4$ for longer distance. In order to conserve energy, senders dynamically adjust the transmission power proportional to the transmission distance. The cost function of transmission is defined as:

$$
C_{R}=\sum_{i=0}^{k-1}\left(P_{T}(i)+P_{R}(i+1)\right)
$$

This selects the route with the minimum cost value. Thus, it can ensure that energy consumption per packet is the minimum. $\mathrm{P}_{\mathrm{T}}$ (i) is proportional to $\| v i$, vi $+1 \| \mathrm{n}$, while $\| v i$, $v i+1 \|$ is the distance between node vi and vi+1. Here, $P_{R}(i+$ 1) can help reduce hop count compared to the original scheme.

\section{B. Remaining Energy Capacity}

This cost metric makes the fairness of energy consumption the main focus. Using remaining energy capacity as an energy metric the energy along the route is calculated as follows:

$$
C_{R}=\sum_{i=1}^{k-1} E_{r}^{i}(t)
$$

The formulae specified above are used for calculation of energies in the algorithm.

\section{Working:}

To incorporate these two metrics AODV algorithm is altered such that the RouteRequest and RouteReply packets sent during route discovery and route table contain fields that provide a measure of the transmitted power and node capacity.

\begin{tabular}{|c|c|c|}
\hline Type & Reserved & Hop Count \\
\hline \multicolumn{3}{|c|}{ RREQ ID } \\
\hline \multicolumn{3}{|c|}{ Destination IP Address } \\
\hline \multicolumn{3}{|c|}{ Destination Sequence Number } \\
\hline \multicolumn{3}{|c|}{ Originator IP Address } \\
\hline \multicolumn{3}{|c|}{ Originator Sequence Number } \\
\hline \multicolumn{3}{|c|}{ Minimum Transmission Power } \\
\hline \multicolumn{3}{|c|}{ Maximum Remaining Energy Capacity } \\
\hline
\end{tabular}

TABLE II: EXTENDED ROUTE REQUEST

\begin{tabular}{|c|c|c|}
\hline Type & Reserved & Hop Count \\
\hline \multicolumn{3}{|c|}{ RREQ ID } \\
\hline \multicolumn{3}{|c|}{ Destination IP Address } \\
\hline \multicolumn{3}{|c|}{ Destination Sequence Number } \\
\hline \multicolumn{3}{|c|}{ Originator IP Address } \\
\hline \multicolumn{3}{|c|}{ Lifetime } \\
\hline \multicolumn{3}{|c|}{ Timestamp } \\
\hline \multicolumn{3}{|c|}{ Minimum Transmission Power } \\
\hline \multicolumn{3}{|c|}{ Maximum Remaining Energy Capacity } \\
\hline
\end{tabular}

TABLE III: EXTENDED ROUTE REPLY

Route Table Entries are as follows:

- Destination IP Address

- Destination Sequence Number

- Valid Destination Sequence Number flag

- Other state and routing flags (e.g., valid, invalid)

- Network Interface

- Hop Count

- Next Hop

- List of Precursors

- Lifetime (expiration or deletion time of the route)

- Maximum Remaining Energy Capacity

- Minimum Transmission Power 
During route discovery from the source to the destination the energy values along the route are accumulated in the RREQ packets. At the destination or intermediate node (which has a fresh enough route to the destination) these values are copied into the RREP packet which is transmitted back to the source. The source alternates between the maximum remaining energy capacity route and minimum transmission route every time it performs route discovery. Each node is initialized with a flag value of 0 .

\section{Algorithm:}

If: flag $==0$

For Route Discovery use Maximum Remaining Energy Capacity Route

Else if: flag $==1$

For Route Discovery use Minimum Total Transmission Power Route

The algorithm once designed is to be evaluated using the performance metrics average energy consumed, convergence time [11], [12], network lifetime, throughtput and packet delivery ratio.

\section{Testing AND RESUlts}

Here five modules average energy consumed, network lifetime, throughput, convergence time and packet delivery ratio are tested for all CEM_AODV, Single Metric AODV and Traditional AODV Protocols. Protocols are tested with four conditions

- Low density and low mobility

- Low density and high mobility

- High density and low mobility

- High density and high mobility

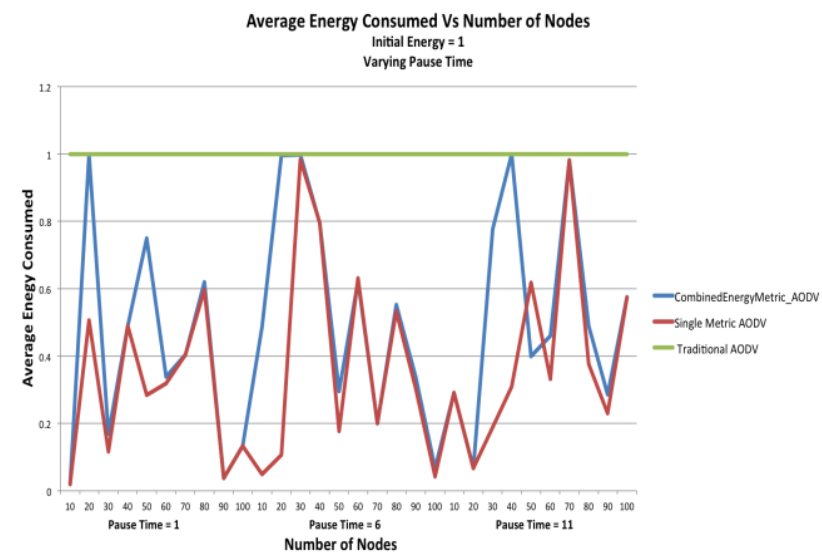

Fig. 1. Average energy consumed Vs number of nodes

Simulations are done for: Number of nodes varying from $[1,100]$ in steps of 10 , Pause Time varying from $[1,50]$ in steps of 5 and Initial Energy Configuration of nodes is 1, 10 and 100 . Thus 10 nodes represent the low node density case, while 100 nodes represent the high node density case. The pause time of 50 implies that the nodes pause in their initial positions for 50 seconds. It represents nodes which have low mobility. Similarly, pause time 0 represents very high mobility where the nodes are in constant motion. Connection Patterns and Mobility Scenarios are kept the same for all three protocols to achieve consistent behaviour. The values are tabulated. At the conclusion of the project, a total of 900 simulations $((100$ scenarios $\times 3$ runs of each scenario $) \times 3$ algorithms) have been run. Test Cases and Graphs have been depicted only for initial energy 1 and 10, pause times 1, 6 and 11 and number of nodes 10 to 100 varying in steps of 10 .

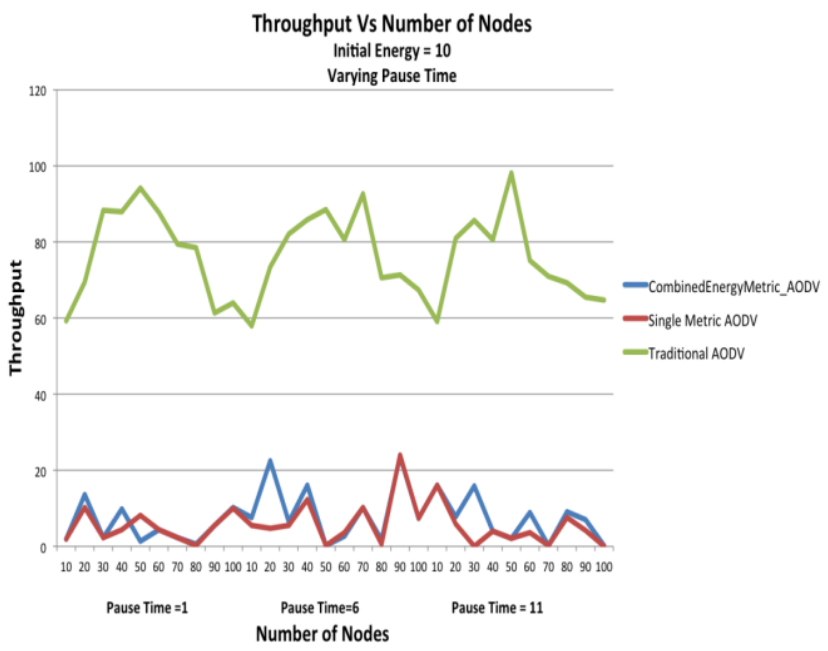

Fig. 2. Throughput Vs number of nodes

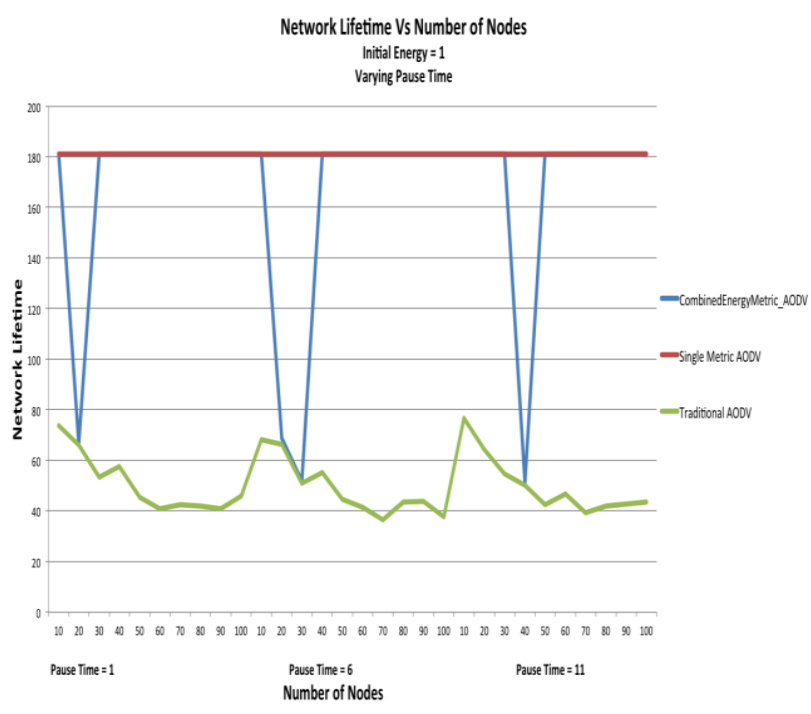

Fig. 3. Network lifetime Vs number of nodes

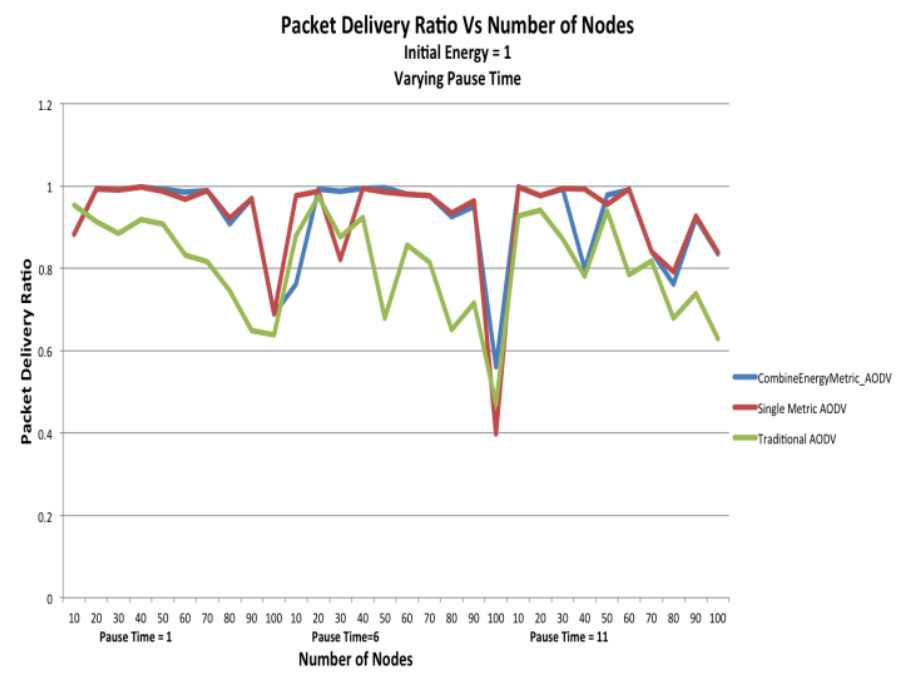

Fig. 4. Packet delivery ratio Vs number of nodes 


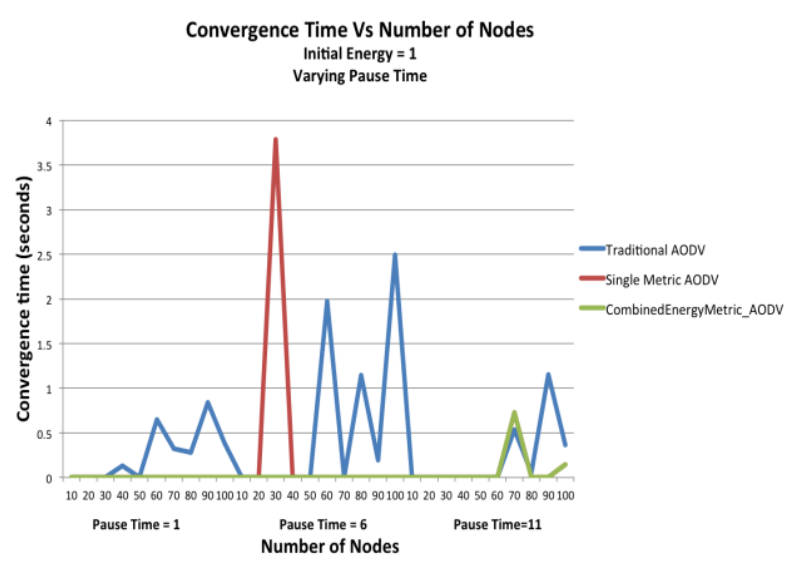

Fig. 5. Convergence time Vs number of nodes

\section{CONCLUSION}

CEM_AODV has better average energy consumed, network lifetime, convergence time and packet delivery ratio than Traditional AODV. But Traditional AODV has better throughput than CEM_AODV.

CEM_AODV has better values of throughput, convergence time and packet delivery ratio than Single Energy Metric AODV. They have the same values for average energy consumed and network lifetime.

\section{FUTURE WORK}

Future work could be to implement caching effectively in reactive protocols as this would allow better energy management. Also, QoS parameters such as throughput and end-to-end delay have to be improved for energy efficient algorithms as these parameters are over looked during design of energy efficient routing protocols.

\section{ACKNOWLEDGMENT}

We would like to express our gratitude to Dr.K Rajanikanth, Principal and Professor, M S Ramaiah Institute of Technology, for providing an environment to work in and for his inspiration during the tenure of the course. We would also like to express our gratitude to Dr. R Selvarani, Professor and Head of Department of Computer Science and Engineering, for her constant support and encouragement.

It is our immense pleasure to express our deep sense of gratitude to Mrs. Annapurna P. Patil, the Project Guide, Associate Professor, Department of Computer Science \& Engineering, for her constant guidance, continual encouragement, understanding, she taught us patience in our endeavour.

\section{REFERENCES}

[1] L. Cao, T. Dahlberg, and Y. Wang, "Performance Evaluation of Energy Efficient Ad Hoc Routing Protocols," IEEE, 2007.

[2] C. Siva Ram Murthy and B. S. Manoj, "Ad Hoc Wireless Networks Architecture and Protocols," $2^{\text {nd }}$ ed, Pearson Education, 2005.

[3] I. Nikolaidis, M. Barbeau, and E. Kranakis, "Ad-Hoc, Mobile, and Wireless Networks," Third International Conference, ADHOC_NOW 2004.

[4] M. Pushpalatha, R. Venkataraman, and T. Ramarao, "Trust Based Energy Aware Reliable Reactive Protocol in Mobile Ad Hoc
Networks," World Academy of Science, Engineering and Technology, 2009.

[5] G. Vijaya Kumar, Y. Vasudeva Reddyr, and M. Nagendra, "Current Research Work on Routing Protocols for MANET: A Literature Survey," (IJCSE) International Journal on Computer Science and Engineering, 2010.

[6] V. Kumar, "Simulation and Comparison of Aodv and Dsr Routing Protocols Inmanets," Master's Thesis, Thapar University(TU), 2009.

[7] R. D. JOSHI and P. P. REGE, "Energy Aware Routing in Ad Hoc Networks,"WSEAS, 2007.

[8] J. Kim and J. Jang, "AODV based Energy Efficient Routing Protocol for Maximum Lifetime in MANET," IEEE, 2006.

[9] L. L. Guo, H. Ma, and T. Jiang, "Energy Efficient on-demand Multipath Routing Protocol for Multi-hop Ad Hoc Networks," IEEE, 2008.

[10] Z. Zhaoxiao, P. Tingrui, and Z. Wenli, "Modified Energy-Aware AODV Routing for Ad hoc Networks," IEEE, 2009.

[11] A. P. Patil, N. Sambaturu, and K. Chunhaviriyakul, "Convergence Time Evaluation of Algorithms in MANETs," International Journal of Computer Science and Information Security, 2009.

[12] A. P. Patil and Harish R, "Performance Evaluation of AODV and AODV+G Algorithms in MANETs Using Convergence Time," in Proc. of (IJCSIS) International Journal of Computer Science and Information Security, vol. 8, no. 5, 2010.

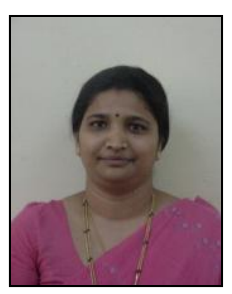

Annapurna P. Patil is a M.Tech,(Ph.D.), Associate Professor, Senior Member IEEE, LMISTE, is a research scholar working at Department of Computer Science and Engineering, M S Ramaiah Institute of Technology, Bangalore, Karnataka, India 560054. She has published several papers in reputed conferences and journals. Her research interests are computer networks and design of intelligent algorithms to solve the computational problems.

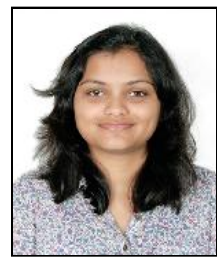

Bathey Sharanya is an Associate Staff Engineer at Oracle India. She has received her B.E degree in Computer Science and Engineering from M.S. Ramaiah Institute of Technology, Bangalore, Karnataka, India 560054 in 2011.

She has published a paper in IJCSI Journal. Her main interests are computer networks, logic design and operating systems.

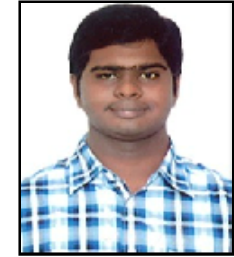

M. P. Dinesh Kumar is a Software Engineer at Aricent Groups. He has received his B.E degree in Computer Science and Engineering from M.S. Ramaiah Institute if Technology, Bangalore, Karnataka, India 560054 in 2011.

He has published a paper in IJCSI Journal. His main interests are Computer Networks, Artificial Intelligence and Operating Systems.

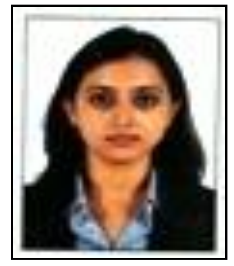

Malavika J. is a Programmer Analyst at Cognizant India. She has received her B.E degree in Computer Science and Engineering from M.S. Ramaiah Institute of Technology, Bangalore, Karnataka, India 560054 in 2011.

She has published a paper in IJCSI Journal. Her main interests are Computer Networks, Information network security and Operating Systems. 